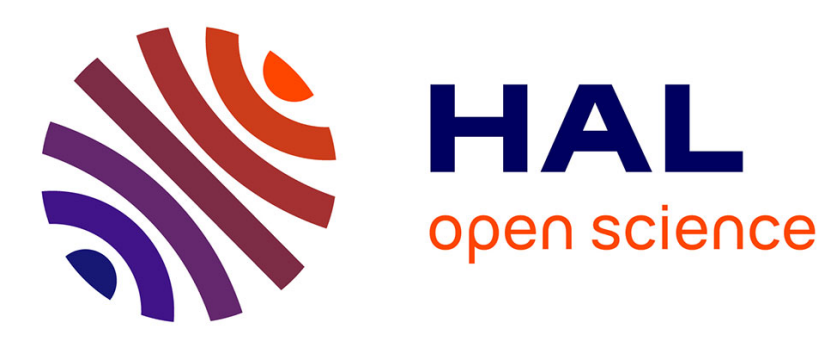

\title{
Formes mathématiques, formes physiques, formes vivantes
}

\author{
Annick Lesne, Julien Mozziconacci
}

\section{To cite this version:}

Annick Lesne, Julien Mozziconacci. Formes mathématiques, formes physiques, formes vivantes. coordonné par A. Fleischer et A. Prochiantz,. Le Rêve de Formes. Arts, Sciences .. et Cie, Le Seuil, pp.107-112, 2019, Le genre humain. hal-02308929

\section{HAL Id: hal-02308929 \\ https://hal.science/hal-02308929}

Submitted on 8 Oct 2019

HAL is a multi-disciplinary open access archive for the deposit and dissemination of scientific research documents, whether they are published or not. The documents may come from teaching and research institutions in France or abroad, or from public or private research centers.
L'archive ouverte pluridisciplinaire HAL, est destinée au dépôt et à la diffusion de documents scientifiques de niveau recherche, publiés ou non, émanant des établissements d'enseignement et de recherche français ou étrangers, des laboratoires publics ou privés. 


\section{Formes mathématiques, formes physiques, formes vivantes}

Annick Lesne ${ }^{1}$ et Julien Mozziconacci ${ }^{2}$

Chaque forme est une interrogation. Les mathématiques y répondent en termes de structures et de symétries, et elles nous offrent un dictionnaire de formes idéales avec lequel décrire notre perception. La physique nous apprend qu'une forme naturelle révèle tout autant les forces et processus qui l'ont engendrée que les propriétés de la matière dans laquelle elle s'incarne. Le vivant ajoute une dimension évolutive et adaptative pour produire des formes fonctionnelles et des organismes autonomes.

Chaque forme est une interrogation. Elle renvoie à une question ancestrale: qu'est-ce que c'est? Est-ce dangereux? L'homme a dû savoir distinguer la forme d'un lion ou celle d'une biche dans une tache beige lointaine. En effet, c'est d'abord et surtout par leur forme que nous identifions les objets qui nous environnent. Celle présentée Figure 1 interpelle ainsi l'observateur, si on ne l'accompagne pas d'explications.

Une première réponse à la question de l'identification d'une forme est apportée par les mathématiques. Les objets mathématiques fournissent un répertoire de formes idéales avec lequel décrire les formes réelles de façon plus ou moins approchée. Par exemple, un cercle et son intérieur, le disque, résument les propriétés géométriques communes à tous les ronds: roue, couronne de pain, rond de serviette, globule rouge. Les formes mathématiques les plus typiques, par exemple le cercle ou le carré, sont associées à des symétries remarquables. La formulation opératoire de la symétrie d'une forme est son invariance par un ensemble de transformations: le cercle est invariant par toute rotation autour de son centre, alors qu'un carré est seulement invariant par rotation d'un ou plusieurs quarts de tour autour de son point central, ou bien par réflexion par rapport à un axe médian. Des symétries plus complexes peuvent également se rencontrer, comme par exemple la symétrie hélicoïdale d'une double hélice (forme mathématique de la molécule d'ADN) ou l'autosimilarité d'une structure fractale (forme mathématique d'un relief côtier ou d'un flocon de neige, invariante sous l'action itérée d'un zoom local).

En général une forme mathématique peut aussi s'exprimer par une équation. Les formes mathématiques présentent ainsi un aspect géométrique (les symétries qu'elles possèdent). Un cercle peut ainsi être défini par l'équation 'rayon = constante', et il est caractérisé (avec le disque et la couronne) par son invariance par toute rotation autour de son centre. Ces formes mathématiques nous permettent de classifier les formes réelles, de percevoir et de décrire les points communs de leur géométrie, en les associant à des archétypes. Ce sont ces formes mathématiques qui sous-tendent l'esquisse d'un objet réel, lorsqu'on se concentre sur sa forme. Elles réduisent la diversité des formes naturelles à un nombre plus restreint de formes abstraites et idéales. En ce sens les formes naturelles apparaissent comme les avatars multiples des formes idéales rencontrées en mathématiques.

\footnotetext{
${ }^{1}$ CNRS, Laboratoire de Physique Théorique de la Matière Condensée, UPMC, CNRS UMR 7600, Sorbonne Université, 4 Place Jussieu, 75252 Paris Cedex 05, France \& Institut de Génétique Moléculaire de Montpellier, University of Montpellier, CNRS, Montpellier, France

${ }^{2}$ Laboratoire de Physique Théorique de la Matière Condensée, UPMC, CNRS UMR 7600, Sorbonne Université, 4 Place Jussieu,75252 Paris Cedex 05, France
} 
Un cercle mathématique est ainsi l'abstraction de tous les ronds matériels. En revanche, il ne dit rien sur la diversité de leurs propriétés physiques, ni sur les mécanismes de leur formation. Il faut pour cela adopter un point de vue physique, dans lequel les formes, qu'elles soient naturelles ou fabriquées, apparaissent comme le lieu de rencontre entre des forces et des matières. Par exemple une goutte d'eau adoptera une forme différente suivant les propriétés de la surface sur laquelle elle est posée, ce qu'on peut observer en regardant la forme des gouttes de rosée sur les feuilles de différentes plantes. Dès qu'on matérialise une forme, les défauts et la granularité de la matière conduisent à une diversité de réalisations, même si le projet en est une forme mathématique idéale. A cette première explication s'ajoute le caractère en partie aléatoire ou contingent des forces à l'œuvre dans la genèse de la forme.

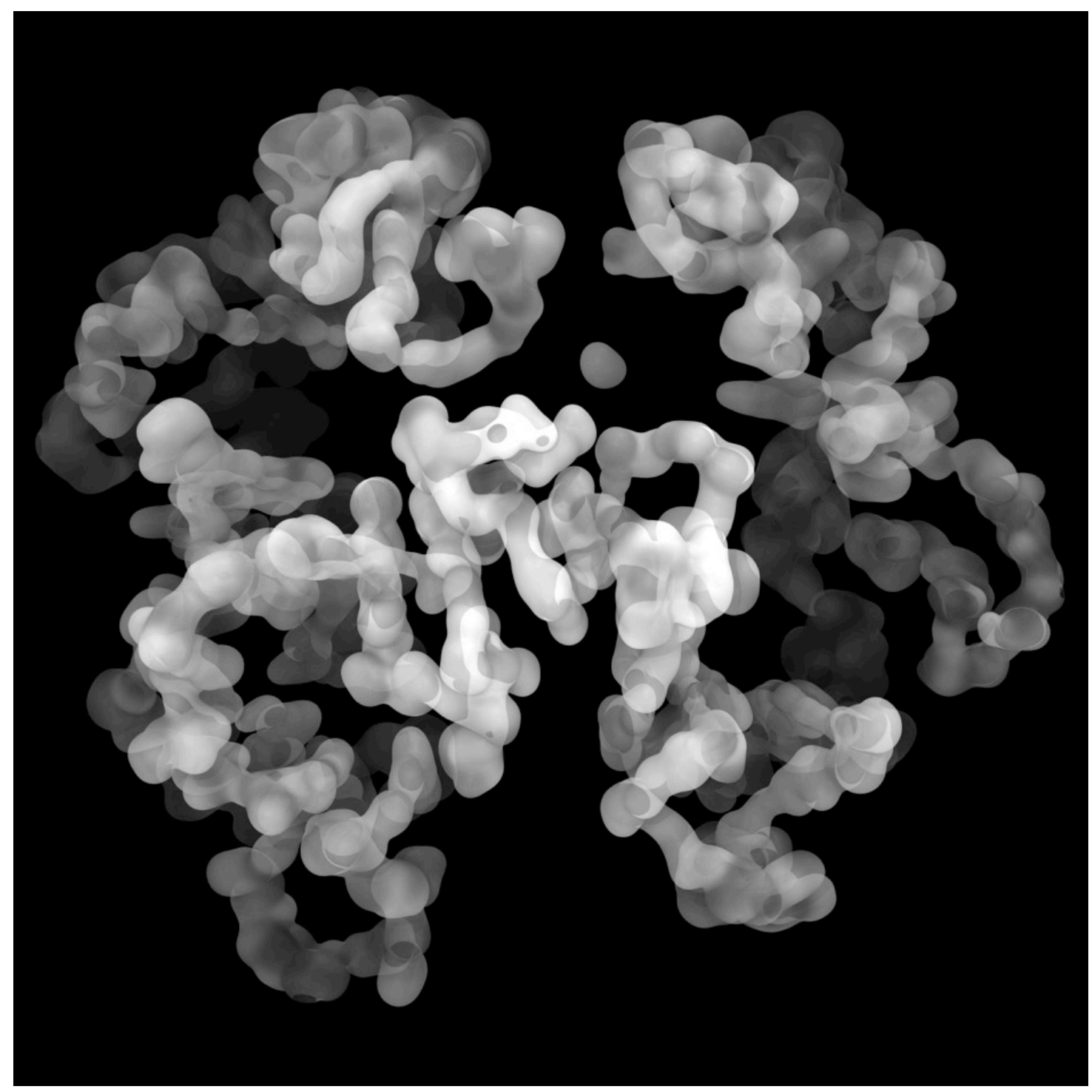

Figure 1 -- Chaque forme est une interrogation: Human Genomics (détail).

Tirage argentique d'une reconstruction numérique à partir de mesures in vivo (2017). Annick Lesne \& Julien Mozziconacci (CNRS \& UPMC), cuvre conçue pour l'exposition Le Rêve des Formes, présentée au Palais de Tokyo du 14 juin au 10 septembre 2017. Production Le Fresnoy-Studio national des arts contemporains avec le soutien de la Fondation Daniel et Nina Carasso. Tirages: Aurélie Brouet et Olivier Anselot; expertise technique: Christophe Gregório. 
Ce point de vue physique sur les formes n'abolit pas la vision mathématique en termes de structures et de symétries, mais il la complète en y incluant des mécanismes de formation et les matières dans lesquelles ils s'inscrivent. Les symétries sont ou bien un guide pour identifier ces mécanismes, ou bien un résultat que l'on peut prédire lorsque ces mécanismes sont déjà connus. Un exemple remarquable est celui des cristaux: les forces microscopiques et l'arrangement atomique qui s'ensuit (formant un réseau cubique à faces centrées, par exemple, pour le sel de cuisine) déterminent la forme des cristaux à toutes les échelles, à l'identique, depuis l'échelle atomique jusqu'à celle que nous voyons à notre échelle macroscopique. Mais c'est une situation assez exceptionnelle, particulière aux cristaux: le plus souvent, les formes qui nous entourent résultent d'une dynamique complexe et de sa mise en œuvre dans des matériaux eux-mêmes hétérogènes, et le lien entre les caractéristiques de leurs constituants et ce que nous observons à l'œil nu est tout sauf évident. Comprendre la conjonction particulière de processus et de matériaux est ainsi nécessaire pour expliquer ou prédire une forme physique. Inversement, l'observation d'une forme concrète révèle beaucoup sur les forces en jeu dans sa formation et sur les matières qui la constituent.

Lorsque leur inscription dans la matière se déploie dans le temps, les formes physiques deviennent capables de raconter des histoires. Les méandres asséchés d'une rivière gardent la mémoire de ses cheminements antérieurs. Les flancs plissés d'une montagne révèlent à la fois le lent dépôt de sédiments et l'émergence du relief sous l'action de forces tectoniques. La forme d'un arbre qui a grandi exposé au vent en indique la direction dominante. Une distorsion de son tronc marque le souvenir d'une agression lors de son développement. La forme d'un figuier étrangleur révèle la présence initiale d'un arbre support, longtemps après que celui-ci ait disparu. Le temps lui-même peut s'imprimer dans une forme lorsqu'elle résulte d'une croissance lente: par exemple, l'histoire d'un coquillage comme une ammonite ou un nautile est encore lisible sur sa coquille, où la distance au centre représente le temps écoulé depuis la naissance.

Une dimension supplémentaire intervient dans les formes vivantes: elles ont une fonction. Par exemple, la fonction de la protéine appelée ARN-polymérase est de transcrire la séquence d'ADN un gène en ARN, celle de l'ADN-polymérase est de dupliquer l'ADN avant la division d'une cellule en deux cellules filles. Cette spécificité des formes vivantes est due à leur évolution par sélection naturelle, qui a posteriori fait apparaître la notion de fonction biologique. Les systèmes biologiques qu'on observe aujourd'hui sont constitués par un ensemble d'éléments que le hasard n'aurait pu assembler sans l'intervention de la sélection naturelle et du filtre qu'elle a réalisé sur le moindre aspect du système, y compris sa forme. La forme d'une organelle, d'une cellule, d'un organe ou même de l'organisme dans son ensemble fait en effet partie des critères qui interviennent dans la sélection naturelle. Les formes vivantes ont donc évolué sous les effets conjuguées du hasard et de la pression de sélection qui s'est exercée sur leurs détails et leurs paramètres, conduisant à une adaptation de ces formes à la fonction qu'elles remplissent dans l'organisme. Une forme vivante contient ainsi l'empreinte de son histoire évolutive.

Ainsi, lorsqu'on cherche l'origine d'une forme vivante, une partie de l'explication est donnée par les lois de la physique, qui régissent les interactions entre ses éléments constitutifs 
(typiquement des macromolécules, en particulier des protéines) complétée par l'intervention répétée de la sélection naturelle, qui a conditionné ces éléments dans les moindres détails. La forme telle qu'elle est observée aujourd'hui résulte d'une coadaptation entre la composition des éléments constitutifs et la mise en œuvre de mécanismes physiques. Pour ces derniers, l'évolution s'est située dans l'ajustement graduel de leurs paramètres: dimensions du système, coefficients des forces en jeu, etc. Une des signatures de l'évolution par sélection naturelle d'un système vivant est ainsi un ajustement fin et robuste de l'ensemble de ses paramètres. On peut même argumenter que la diversité des formes biologiques va de pair avec l'implication de mécanismes physiques, qui eux sont fondés sur des lois universelles. C'est précisément l'adaptation à des principes de fonctionnement généraux, dans toutes les conditions particulières que rencontre un organisme vivant, qui exige l'existence de formes particulières à chaque organisme.

Est-on capable, par le simple regard porté sur l'image d'une forme, de distinguer s'il s'agit d'une forme mathématique, d'une forme physique ou d'une forme vivante? La perfection des formes mathématiques et le caractère fonctionnel des formes biologiques peuvent guider l'intuition, mais ce n'est pas toujours aussi simple. En particulier, les mathématiques sont également capables de décrire et d'engendrer à l'aide d'équations des structures aléatoires, d'apparence désordonnée. Un exemple typique est la trajectoire d'un mouvement Brownien, idéalisation mathématique du mouvement d'un grain de pollen en suspension dans l'eau, qui suit une trajectoire erratique sous l'effet d'innombrables collisions avec les mouvements aléatoires des molécules d'eau en agitation thermique. De nombreuses autres sources de hasard et de contingence interviennent dans la genèse des formes naturelles. Dans le vivant, elles sont en partir canalisées par la sélection naturelle et la régulation à l'œuvre dans les processus biologiques, permettant de reproduire assez fidèlement la forme des organismes, plus fidèlement en tous cas que la nature répète la forme d'une montagne ou d'une stalactite.

Revenons à l'image initiale, un extrait de l'œuvre Human Genomics présentée dans l'exposition le Rêve des Formes, au Palais de Tokyo durant l'été 2017. Elle représente le chromosome 1, le plus grand de nos chromosomes (numérotés par ordre de taille décroissante). Nos chromosomes ont une structure interne remarquablement complexe, hiérarchique, présentant de nombreux niveaux d'organisation successifs depuis le niveau de l'ADN jusqu'à celui du chromosome dans son ensemble. Le filament sur notre image n'est pas l'ADN mais une superstructure, la fibre de chromatine. Cette fibre implique l'ADN de notre génome mais aussi des protéines autour desquelles l'ADN s'enroule, et elle va elle-même se replier dans l'espace. Il n'est pas simple d'observer le chromosome dans des conditions physiologiques. La microscopie ne permet qu'une visualisation biaisée par la préparation de l'échantillon (coupe, fixation, insertion de molécules fluorescentes). Les observations les plus fiables qu'il est possible d'effectuer aujourd'hui sont indirectes, à l'aide d'une technique appelée «capture de conformations chromosomiques». Son principe est de réaliser un pontage chimique des régions de l'ADN se trouvant proches l'une de l'autre dans l'espace, suivi d'étapes de coupure, de ligature et de séquençage des régions impliquées dans ces pontages. Ce protocole expérimental donne ainsi accès à la fréquence avec laquelle deux régions du chromosome se trouvent en contact physique à l'intérieur des noyaux cellulaires. On voit assez facilement que la forme du chromosome détermine l'ensemble des fréquences 
de contact entre ses différentes régions, mais le problème inverse, à savoir reconstituer la forme à partir de la mesure des fréquences de contact, n'a rien d'évident. Nous avons dans ce but développé un algorithme $e^{3}$ impliquant une suite de transformations mathématiques des fréquences de contact. Il permet d'obtenir les coordonnées en trois dimensions du chromosome, autrement dit sa forme explicite, que l'on peut ensuite visualiser à l'aide de logiciels graphiques. Le fichier numérique obtenu a enfin été transformé en négatif argentique et un tirage photographique en a été réalisé. L'image n'est donc pas directement une photographie du chromosome, mais une reconstruction numérique.

Pourquoi s'intéresser à la forme tridimensionnelle de nos chromosomes? Tout simplement parce qu'elle a un rôle fonctionnel, bien plus complexe que seulement assurer que les très longues molécules d'ADN formant nos chromosomes (au total de l'ordre de plusieurs mètres) rentrent dans l'espace très réduit du noyau de nos cellules (quelques microns de diamètre, soit un million de fois moins). Le repliement du génome dans l'espace participe en particulier à la régulation de l'expression des gènes, en mettant en contact différentes régions du génome et les protéines qui y sont attachées. Cette proximité spatiale leur permet d'interagir et de réaliser les différentes étapes de l'expression des gènes situés à cet endroit. La forme d'un chromosome à l'intérieur du noyau de nos cellules résulte finalement de l'interaction entre une matière, la chromatine, et de tous les processus fonctionnels impliquant le génome, finement et graduellement mise en place au cours de l'évolution du vivant. Inversement, cette forme reflète le rôle fonctionnel que le chromosome remplit aujourd'hui. Par les fonctions qu'elle assure, cette forme va déterminer le développement de l'organisme, dans l'espace et le temps: indirectement, la forme du chromosome contribue ainsi à la forme de l'organisme, dans une dynamique spatio-temporelle complexe qui est loin d'être entièrement élucidée.

On comprend alors la particularité de la forme montrée Figure 1: il s'agit d'une forme hybride, parce qu'il ne s'agit pas d'une photo, ni d'une sculpture, ni d'un dessin, mais d'une reconstruction mathématique à partir de mesures physiques d'un objet biologique.

\footnotetext{
${ }^{3}$ Les lecteurs intéressés peuvent trouver les détails de cet algorithme, appelé ShRec3D (pour Shortestpath Reconstruction in 3D), dans Lesne, Riposo, Roger, Cournac \& Mozziconacci, Nature Methods vol. 11, pp. 1141-1143 (2014).
} 